Mathematical Modelling and AnAlysis

Volume 18 Number 3, June 2013, 395-414

http://dx.doi.org/10.3846/13926292.2013.804891

(c) Vilnius Gediminas Technical University, 2013
Publisher: Taylor\&Francis and VGTU

http://www.tandfonline.com/TMMA

Print ISSN: 1392-6292

Online ISSN: 1648-3510

\title{
A Strongly Ill-Posed Integro-Differential Parabolic Problem with Integral Boundary Conditions*
}

\author{
Alfredo Lorenzi \\ Università degli Studi di Milano \\ via Saldini 50, 20133 Milano, Italy \\ E-mail: alfredo.lorenzi@mat.unimi.it
}

Received 16 October, 2012; revised April 26, 2013; published online June 1, 2013

\begin{abstract}
Via Carleman estimates we prove uniqueness and continuous dependence results for an identification and strongly ill-posed linear integro-differential parabolic problem with the Dirichlet boundary condition, but with no initial condition. The additional information consists in a boundary linear integral condition involving the normal derivative of the temperature on the whole of the lateral boundary of the space-time domain.
\end{abstract}

Keywords: ill-posed problems, linear parabolic integro-differential equations, no initial condition, integral boundary conditions, uniqueness, continuous dependence results.

AMS Subject Classification: primary 35R30; secondary 35K20, 45K05, 45Q05.

\section{Introduction}

This paper is concerned with the determination of an unknown time-dependent function in a strongly ill-posed problem, where strongly means that no transformation can be found in order to change our problem to a well-posed one, at least, when working in classical or Sobolev function spaces of finite order.

We stress that the main stream concerning strongly ill-posed problems involves PDE's. For this purpose we refer the reader to the monograph [4], dealing with elliptic and parabolic problems, and the rich bibliography therein. Of course, lesser interest was devoted to recovering unknown functions in strongly ill-posed integro-differential problems. This paper is just devoted to shed some light on such problems, mainly on the questions of uniqueness and continuous dependence on the data, two fundamental topics for people working in Applied Mathematics. More exactly, we will deal here with an integro-differential linear parabolic problem, where in the integral operator the integrations involve both

\footnotetext{
* A. Lorenzi is a member of G.N.A.M.P.A. of the Italian Istituto Nazionale di Alta Matematica (I.N.d.A.M).
} 
space and time and the operator is of Volterra type. Furthermore in our problem no initial condition will be supplied. It will be replaced by the requirement that the "temperature" $u$ should assume prescribed values on $(0, T) \times \partial \Omega$ as well as an additional boundary condition involving a linear combination of the flux of $u$ and a Volterra type integral of the temperature itself will be prescribed on $(0, T) \times \partial \Omega$.

The main task of this paper consists in recovering a function $\alpha$ in the source term when a mean of the temperature is known for all times $t \in[0, T]$. Moreover, we shall be able to estimate $u$ in $C\left((0, T] ; L^{2}(\Omega)\right) \cap L_{l o c}^{2}\left((0, T] ; H^{1}(\Omega)\right)$ in terms of suitable norms of our data and $\alpha$ in $L^{2}((0, T] ; \mathbb{R})$.

The fundamental tool to give a positive answer to our problem will be deduced by adapting to our case the celebrated (weighted) Carleman estimates for PDE's [5,6] - of use both in Control and Inverse Problem Theory. They will ensure the uniqueness of the solution to our problem and also an unusual (weighted) result involving continuous dependence on the data. To improve this result to one related to usual $L^{2}$-spaces we shall add a few new assumptions on the kernels and some additional analysis.

We conclude this introduction by observing that, although there is a wide literature concerned with the problem of recovering an unknown function entering a well-posed parabolic problem and also a rich literature dealing with ill-posed problems for PDE's, at the best of our knowledge in these last years no paper can be found in MathScinet corresponding to the keywords ill posed and identification or recovering and concerned with integro-differential equations with integral boundary conditions. We quote also a few related papers concerning the identification of constants in strongly ill-posed parabolic problems $[8,9]$ or in well-posed ones $[1,3,7,10,11,12,13]$.

The plan of the paper is the following: Section 2 reports Carleman estimates for linear second-order parabolic equations when the additional information is given on the lateral boundary. In Section 3 we express the unknown function as an affine operator involving $u$, so that we can transform the given integro-differential identification problem into a strongly (non-standard) illposed integro-differential problem for $u$. In Section 4 we determine sufficient conditions on our data and the integral kernels leading to a Carleman estimate of weak type, i.e. with a fixed parameter $s_{0}$ (cf. Theorem 1), as well as we deduce the desired weighted $L^{2}$-estimate for unknown function $\alpha$. Finally, in Section 5 we determine sufficient conditions on the integral kernels ensuring the continuous dependence on our data of $(u, \alpha)$ in the non-weighted spaces $\left[C\left((0, T] ; L^{2}(\Omega)\right) \cap L_{l o c}^{2}\left((0, T] ; L^{2}(\Omega)\right)\right] \times L^{2}((0, T] ; \mathbb{R})(\mathrm{cf}$. Theorem 2$)$. 


\section{An Auxiliary Linear Strongly Ill-Posed Problem}

Let $\Omega$ be a bounded open sets in $\mathbb{R}^{n}, \partial \Omega$ being of $C^{2}$-class. Let $A(x, D)$ be the (formal) uniformly elliptic linear operator defined by

$$
\begin{aligned}
A(x, D) & =\sum_{i, j=1}^{n} D_{x_{i}}\left[a_{i, j}(x) D_{x_{j}}\right]+\sum_{j=1}^{n} D_{x_{j}}\left[a_{j}(x) \cdot\right] \\
& =: A_{1}(x, D)+\sum_{j=1}^{n} D_{x_{j}}\left[a_{j}(x) \cdot\right]
\end{aligned}
$$

where

$$
\begin{aligned}
& a_{i, j} \in C^{1}(\bar{\Omega}), \quad a_{j, i}=a_{i, j}, \quad a_{j} \in W^{1, \infty}(\Omega), \quad i, j=1, \ldots n, \\
& \mu_{0}|\xi|^{n} \leq \sum_{i, j=1}^{n} a_{i, j}(x) \xi_{i} \xi_{j} \leq \mu_{1}|\xi|^{n}, \quad x \in \bar{\Omega}, \quad \xi \in \mathbb{R}^{n},
\end{aligned}
$$

for some positive constants $\mu_{0}$ and $\mu_{0}, \mu_{0} \leq \mu_{1}$.

We consider the following ill-posed problem, where $Q_{T}=(0, T) \times \Omega$ and $\Sigma_{T}=(0, T) \times \partial \Omega$ :

$$
\begin{cases}u \in H^{1}\left((0, T) ; L^{2}(\Omega)\right) \cap L^{2}\left((0, T) ; H^{2}(\Omega)\right), & \\ D_{t} u(t, x)-A(x, D) u(t, x)=q(t, x), & (t, x) \in Q_{T}, \\ u(t, x)=g_{0}(t, x), & (t, x) \in \Sigma_{T} \\ D_{\nu} u(t, x)=g_{1}(t, x), & (t, x) \in \Sigma_{T}\end{cases}
$$

Here $D_{\nu}$ denotes the (outer) normal derivative, $\nu$ denoting the (outer) normal vector-field. Moreover, we assume

$$
q \in L^{2}\left(Q_{T}\right), \quad g_{0} \in H^{1}\left((0, T) ; L^{2}(\Omega)\right) \cap L^{2}\left((0, T) ; H^{2}(\Omega)\right) .
$$

Then the function $v=u-g_{0}$ solves the (equivalent) ill-posed problem

$$
(\mathrm{IP} 2) \begin{cases}v \in H^{1}\left((0, T) ; L^{2}(\Omega)\right) \cap L^{2}\left((0, T) ; H^{2}(\Omega)\right), & \\ D_{t} v(t, x)-A(x, D) v(t, x) & \\ \quad=q(t, x)-D_{t} g_{0}(t, x)+A(x, D) g_{0}(t, x), & (t, x) \in Q_{T}, \\ v(t, x)=0, & (t, x) \in \Sigma_{T} \\ D_{\nu} v(t, x)=g_{1}(t, x)-D_{\nu} g_{0}(t, x), & (t, x) \in \Sigma_{T} .\end{cases}
$$

In the sequel we shall need the Carleman estimate related to problem (IP2). For this purpose we begin by introducing the functions $\varphi_{\lambda}: \bar{\Omega} \rightarrow \mathbb{R}, \alpha_{\lambda}$ : $[0, T] \times \bar{\Omega} \rightarrow \mathbb{R}$, depending on the parameter $\lambda \in[1,+\infty)$, defined by

$$
\varphi_{\lambda}(t, x)=e^{\lambda \psi(x)}, \quad \alpha_{\lambda}(t, x)=\frac{e^{\lambda \psi(x)}-e^{2 \lambda\|\psi\|_{\infty}}}{l(t)}, \quad(t, x) \in(0, T) \times \bar{\Omega},
$$

where

$$
l(t)=t(T-t)
$$


and function $\psi \in C^{4}(\bar{\Omega})$ satisfies the properties (cf. [5])

$$
\psi(x)>0, x \in \Omega, \quad|\nabla \psi(x)| \geq \mu_{2}>0, x \in \bar{\Omega}, \quad D_{\nu_{A}} \psi(x) \leq 0, x \in \partial \Omega \backslash \Gamma
$$

for some positive constant $\mu_{2}$.

Owing to Lemma 2.4 , with $p=0$, in [6], since $\varphi_{\lambda}(x) \geq 1$ for all $x \in \bar{\Omega}$, any solution $v \in H^{1}\left((0, T) ; L^{2}(\Omega)\right) \cap L^{2}\left((0, T) ; H^{2}(\Omega) \cap H^{1}(\Omega)\right)$ to problem (IP2) satisfies the Carleman estimate

$$
\begin{aligned}
& s^{3} \int_{Q_{T}} l(t)^{-3}|v(t, x)|^{2} \exp \left[2 s \alpha_{\lambda}(t, x)\right] d t d x \\
& +s \int_{Q_{T}} l(t)^{-1}|\nabla v(t, x)|^{2} \exp \left[2 s \alpha_{\lambda}(t, x)\right] d t d x+s^{-1} e^{-\lambda \psi_{m}} \\
& \quad \times \int_{Q_{T}} l(t)\left[\left|D_{t} v(t, x)\right|^{2}+\sum_{i, j=1}^{n}\left|D_{x_{i}} D_{x_{j}} v(t, x)\right|^{2}\right] \exp \left[2 s \alpha_{\lambda}(t, x)\right] d t d x \\
& \leq s^{3} \int_{Q_{T}} l(t)^{-3} \varphi_{\lambda}(x)^{3}|v(t, x)|^{2} \exp \left[2 s \alpha_{\lambda}(t, x)\right] d t d x \\
& +s \int_{Q_{T}} l(t)^{-1} \varphi_{\lambda}(x)|\nabla v(t, x)|^{2} \exp \left[2 s \alpha_{\lambda}(t, x)\right] d t d x \\
& +s^{-1} \int_{Q_{T}} l(t) \varphi_{\lambda}(x)^{-1}\left[\left|D_{t} v(t, x)\right|^{2}+\sum_{i, j=1}^{n}\left|D_{x_{i}} D_{x_{j}} v(t, x)\right|^{2}\right] \\
& \quad \times \exp \left[2 s \alpha_{\lambda}(t, x)\right] d t d x \\
& \leq C_{1}\left\{\int_{Q_{T}}\left[\left|D_{t} g_{0}(t, x)\right|^{2}+\left|A(x, D) g_{0}(t, x)\right|^{2}\right] \exp \left[2 s \alpha_{\lambda}(t, x)\right] d t d x\right. \\
& +\left.\int_{\mid} h(t, x)\right|^{2} \exp \left[2 s \alpha_{\lambda}(t, x)\right] d t d x \\
& \left.+s \int_{\Sigma_{T}} l(t)^{-1} \varphi_{\lambda}(x) \exp \left[2 s \alpha_{\lambda}(t, x)\right]\left|D_{\nu} v(t, x)\right|^{2} d t d x\right\}, \quad s \geq \widehat{s}_{0} . \\
& +
\end{aligned}
$$

Here $|\psi|_{\infty}=\min _{x \in \bar{\Omega}} \psi(x)$ and the positive constants $C_{1}, \lambda$ and $\widehat{s}_{0}$ depend on $\mu_{0}, T,\left\|a_{i, j}\right\|_{L^{\infty}(\Omega)},\left\|a_{j}\right\|_{W^{1, \infty}(\Omega)}, i, j=1, \ldots, n$, and $\Omega$.

\section{The Strongly Ill-Posed Identification Problem}

We consider the ill-posed problem consisting in recovering the function $\alpha$ : $(0, T) \rightarrow \mathbb{R}$ in the linear integro-differential parabolic problem

$$
\begin{cases}u \in H^{1}\left((0, T) ; L^{2}(\Omega)\right) \cap L^{2}\left((0, T) ; H^{2}(\Omega)\right), & \\ D_{t} u(t, x)-A(x, D) u(t, x) & (t, x) \in Q_{T}, \\ \quad=B_{1} u(t, x)+\alpha(t) f(t, x)+h(t, x), & (t, x) \in \Sigma_{T}, \\ u(t, x)=0, & (t, x) \in \Sigma_{T},\end{cases}
$$


$\nu$ denoting the (outer) normal to $\partial \Omega$, under the following additional condition, standing for an (integral) spatial mean of $u$ :

$$
\int_{\Omega} u(t, x) d x=\beta(t), \quad t \in(0, T) .
$$

Here $\beta:(0, T) \rightarrow \mathbb{R}$ and $B_{1}$ and $B_{2}$ are the linear integral operators defined by

$$
\begin{aligned}
& B_{1} w(t, x)=\int_{Q_{t}} k_{1}(t, x, r, y) w(r, y) d r d y, \quad(t, x) \in Q_{T}, \\
& B_{2} w(t, x)=\int_{Q_{t}} k_{2}(t, x, r, y) w(r, y) d r d y, \quad(t, x) \in \Sigma_{T},
\end{aligned}
$$

where the kernels $k_{j}: E \rightarrow \mathbb{R}$, with $j=1,2$ and $E_{1}=\left\{(t, x, r, y) \in Q_{T} \times Q_{T}\right.$ : $r<t\}$ and $E_{2}=\left\{(t, x, r, y) \in \Sigma_{T} \times Q_{T}: r<t\right\}$, are measurable and, for the time being, separably integrable over $Q_{T}$, if $j=1$, and over $\Sigma_{T}$, if $j=2$, with respect to $(t, x)$ and over $Q_{T}$ with respect to $(r, y)$.

We note that, up to a translation, a problem with a non-vanishing $u$ on $\Sigma_{T}$, can be reduced to one with a vanishing $u$ on $\Sigma_{T}$.

We need now to compute the conormal derivative $D_{\nu_{A}} u$ in terms of the normal derivative $D_{\nu} u$. For this task we recall that the conormal vector-field $\nu_{A_{1}}$ is defined by

$$
\left(\nu_{A}\right)_{i}=\sum_{j=1}^{n} a_{i, j}(x) \nu_{i}(x), \quad x \in \partial \Omega, i=1, \ldots, n .
$$

Then we note that, if $\tau^{(j)}(x), j=1, \ldots, d-1$, denote the set of $d-1$ unit vectors that are mutually orthogonal and tangent to $\partial \Omega$ at $x$, we have

$$
\nu_{A}(x)=\sum_{j=1}^{d-1} c_{j}(x) \tau^{(j)}(x)+\left[\nu_{A_{1}}(x) \cdot n(x)\right] \nu(x), \quad x \in \partial \Omega .
$$

Since

$$
\bar{\nu}_{A}(x):=\nu_{A}(x) \cdot n(x)=\sum_{j=1}^{d} a_{i, j}(x) n_{i}(x) n_{j}(x) \geq \mu_{0}>0, \quad \text { for all } x \in \partial \Omega
$$

and all the tangential derivative of $u$ vanish according to the first boundary condition in (3.1), we easily deduce the relation

$$
D_{\nu_{A}} u(x)=\bar{\nu}_{A}(x) D_{\nu} u(x), \quad x \in \partial \Omega .
$$

Apply now the functional $J w=\int_{\Omega} w(x) d x$ to both sides of the differential equation in (IP2) and consider the following formulae (cf. (2.2)):

$$
\begin{aligned}
\int_{\Omega} A u(t, x) d x & =\int_{\partial \Omega} D_{\nu_{A}} u(t, x) d \sigma(x)=\int_{\partial \Omega} \bar{\nu}_{A}(x) D_{\nu} u(t, x) d \sigma(x) \\
& =\int_{\partial \Omega} \bar{\nu}_{A}(x) g(t, x) d \sigma(x)+\int_{\partial \Omega} \bar{\nu}_{A}(x) B_{2} u(t, x) d \sigma(x),
\end{aligned}
$$


where $\sigma$ denotes the Lebesgue surface measure.

Consider now the identities

$$
\begin{aligned}
\int_{\Omega} B_{1} u(t, x) d x & =\int_{\Omega} d x \int_{(0, t) \times \Omega} k_{1}(t, x, r, y) u(r, y) d r d y \\
& =\int_{(0, t) \times \Omega} \widetilde{k}_{1}(t, r, y) u(r, y) d r d y \\
\int_{\partial \Omega} \bar{\nu}_{A}(x) B_{2} u(t, x) d \sigma(x) & =\int_{\partial \Omega} \bar{\nu}_{A}(x) d \sigma(x) \int_{(0, t) \times \Omega} k_{2}(t, x, r, y) u(r, y) d r d y \\
& =\int_{(0, t) \times \Omega} \widetilde{k}_{2}(t, r, y) u(r, y) d r d y,
\end{aligned}
$$

where

$$
\begin{aligned}
& \widetilde{k}_{1}(t, r, y)=\int_{\Omega} k_{1}(t, x, r, y) d x \\
& \widetilde{k}_{2}(t, r, y)=\int_{\partial \Omega} \bar{\nu}_{A}(x) k_{2}(t, x, r, y) d \sigma(x) .
\end{aligned}
$$

We easily deduce the equation for $\alpha$ :

$$
-\int_{Q_{t}} \widetilde{k}(t, r, x) u(r, y) d r d y+\gamma(\beta, f)(t)=\alpha(t) \int_{\Omega} f(t, x) d x,
$$

where

$$
\begin{aligned}
& \widetilde{k}(t, r, y)=\sum_{j=1}^{2} \widetilde{k}_{j}(t, r, y), \\
& \gamma(\beta, g, h)(t)=\beta^{\prime}(t)-\int_{\partial \Omega} \nu_{A}(x) g(t, x) d \sigma(x)-\int_{\Omega} h(t, x) d x .
\end{aligned}
$$

Assume now

$$
\left|\int_{\Omega} f(t, x) d x\right| \geq m>0, \quad \forall t \in[0, T]
$$

and set $\chi(f)(t)^{-1}:=\int_{\Omega} f(t, x) d x$. Then $\alpha$ can be represented as an operator of $u$ :

$$
\begin{aligned}
\alpha(t) & =-\chi(f)(t) \int_{(0, t) \times \Omega} \widetilde{k}(t, r, y) u(r, y) d r d y+\chi(f)(t) \gamma(\beta, g, h)(t) \\
& =:-\Lambda(u)(t)-I(\beta, f, g, h)(t) .
\end{aligned}
$$

Consequently, $u$ has to solve the strongly ill-posed integro-differential problem

$$
\begin{cases}u \in H^{1}\left((0, T) ; L^{2}(\Omega)\right) \cap L^{2}\left((0, T) ; H^{2}(\Omega)\right), & \\ D_{t} u(t, x)-A(x, D) u(t, x) & (t, x) \in Q_{T}, \\ \quad=\mathcal{B} u(t, x)+\widetilde{h}(t, x), & (t, x) \in \Sigma_{T}, \\ u(t, x)=0, & (t, x) \in \Sigma_{T}, \\ D_{n} u(t, x)=g(t, x)+B_{2} u(t, x), & \end{cases}
$$


where

$$
\begin{aligned}
\mathcal{B} u(t, x) & =B_{1} u(t, x)-\Lambda(u)(t) f(t, x), \\
\widetilde{h}(t, x) & =h(t, x)-I(\beta, f, g, h)(t) f(t, x), \quad(t, x) \in Q_{T} .
\end{aligned}
$$

\section{An Estimate of Weak Carleman Type}

Let $u$ be a solution to problem (IP4). Then $u$ solves problem (IP2) with $q=\mathcal{B}+\widetilde{h}, g_{0}=0$, and $g_{1}=g+B_{2} u$. Consequently, from (2.5), with $s=s_{0} \geq \widehat{s}_{0}$ to be chosen later on, we deduce the following estimate

$$
\begin{array}{rl}
\int_{Q_{T}} & l(t)^{-3}|u(t, x)|^{2} \exp \left[2 s_{0} \alpha_{\lambda}(t, x)\right] d t d x \\
& +s_{0} \int_{Q_{T}} l(t)^{-1}|\nabla u(t, x)|^{2} \exp \left[2 s_{0} \alpha_{\lambda}(t, x)\right] d t d x+s_{0}^{-1} e^{-\lambda\|\psi\|_{\infty}} \\
& \times \int_{Q_{T}} l(t)\left[\left|D_{t} u(t, x)\right|^{2}+\sum_{i, j=1}^{n}\left|D_{x_{i}} D_{x_{j}} u(t, x)\right|^{2}\right] \exp \left[2 s_{0} \alpha_{\lambda}(t, x)\right] d t d x \\
\leq & 2 C_{1} \int_{Q_{T}}|\mathcal{B} u(t, x)|^{2} \exp \left[2 s_{0} \alpha_{\lambda}(t, x)\right] d t d x \\
& +2 C_{1} \int_{Q_{T}}|\widetilde{h}(t, x)|^{2} \exp \left[2 s_{0} \alpha_{\lambda}(t, x)\right] d t d x \\
& +2 s_{0} C_{1} \int_{\Sigma_{T}}\left[|g(t, x)|^{2}+\left|B_{2} u(t, x)\right|^{2}\right] \exp \left[2 s_{0} \alpha_{\lambda}(t, x)\right] d t d \sigma(x)
\end{array}
$$

To prove our weak Carleman estimate, ${ }^{1}$ related to a suitable $s_{0}$, for our illposed integro-differential problem (IP4) we need four positive constants $K_{j}$, $j=0, \ldots, 3$, and choose $s_{0}$ satisfying the inequalities

$$
p_{0}\left(s_{0}, f\right):=s_{0}\left[s_{0}^{2}-2 C_{1} K_{4}(f)\right]>0, \quad s_{0} \geq \widehat{s}_{0}, \quad s_{0} \geq 1
$$

where

$$
K_{4}(f)=K_{0} K_{1}+m^{-2}\|f\|_{L^{\infty}\left(Q_{T}\right)}^{2} m(\Omega)\left[K_{0}+\mu_{1} K_{2}\right]\left[K_{1}+\mu_{1} K_{3}\right]>0 .
$$

Once we have fixed $s_{0}$, we are allowed to make the following assumptions concerning $k_{1}$ and $k_{2}$ :

$$
\begin{aligned}
& \int_{Q_{t}}\left|k_{j}(t, x, r, y)\right| d r d y \leq K_{2(j-1)} \\
& \quad(t, x) \in Q_{T}, \text { if } j=1, \quad(t, x) \in \Sigma_{T}, \text { if } j=2, \\
& \int_{(r, T) \times F_{j}}\left|k_{j}(t, x, r, y)\right| d t d \rho_{j}(x) \leq K_{2 j-1} l(r)^{-3} \exp \left[-2 s_{0} c_{1}(\psi) l(r)^{-1}\right], \\
& \quad(r, y) \in Q_{T}, j=1,2, F_{1}=\Omega, F_{2}=\partial \Omega, \quad \rho_{1}=m, \rho_{2}=\sigma
\end{aligned}
$$

$m$ and $\sigma$ denoting, respectively, the $n$-dimensional Lebesgue measure and the related Lebesgue surface measure.

\footnotetext{
${ }^{1}$ Weak means here that the Carleman estimate holds for a fixed $s=s_{0}$.
} 
Remark 1. To exhibit an example of functions $k_{j}, j=1,2$, satisfying conditions (4.4), (4.5) we choose the functions

$$
k_{j}(t, x, r, y)=\sum_{i=1}^{+\infty} h_{1, i, j}(t, x) h_{2, i, j}(r, y), \quad j=1,2,
$$

where the functions $h_{1, i, j} \in L^{\infty}\left((0, T) \times F_{j}\right)$ and $h_{2, i, j} \in L^{\infty}\left(Q_{T}\right)$ satisfy the following bounds for all $i \in \mathbb{N}, j=1,2$ :

$$
\begin{aligned}
& \left|h_{2, i, j}(r, y)\right| \leq \kappa_{i, j} l(t)^{-3} \exp \left[-2 s_{0} c_{1}(\psi) l(t)^{-1}\right], \quad(r, y) \in Q_{T}, j=1,2, \\
& \sum_{i=1}^{+\infty} \kappa_{i, j}\left\|h_{1, i, j}\right\|_{L^{\infty}\left((0, T) \times F_{j}\right)}<+\infty, \quad j=1,2,
\end{aligned}
$$

for some nonnegative constants $\kappa_{i, j}, i \in \mathbb{N}, j=1,2$. Indeed, assumptions (4.7)-(4.8) imply

$$
\begin{aligned}
& \int_{Q_{T}} \mid k_{j}(t, x, r, y)\left|d r d y \leq \sum_{i=1}^{\infty}\right| h_{1, i, j}(t, x) \mid\left\|h_{2, i, j}\right\|_{L^{1}\left(Q_{T}\right)} \\
& \leq 4 m(\Omega) \sum_{i=1}^{\infty} \kappa_{i, j}\left\|h_{1, i, j}\right\|_{L^{\infty}\left((0, T) \times F_{j}\right)} \\
& \times \int_{4 / T^{2}}^{\infty} s^{3 / 2}\left(T^{2} s-4\right)^{-1 / 2} \exp \left[-2 s_{0} c_{1}(\psi) s\right] d s=: K_{2(j-1)}, \quad j=1,2 \\
& \int_{(r, T) \times F_{1}}\left|k_{1}(t, x, r, y)\right| d t d x \leq \sum_{i=1}^{\infty}\left\|h_{1, i, j}\right\|_{L^{1}\left((0, T) \times F_{1}\right)}\left|h_{2, i, j}(r, y)\right| \\
& \leq \frac{27}{8}\left[c_{1}(\psi)\right]^{-3} m(\Omega) \sum_{i=1}^{\infty} \kappa_{i, j}\left\|h_{1, i, j}\right\|_{L^{\infty}((0, T) \times \Omega)}=: K_{1}, \\
& \int_{(r, T) \times F_{2}}\left|k_{2}(t, x, r, y)\right| d t d \sigma(x) \leq \sum_{i=1}^{\infty}\left\|h_{1, i, j}\right\|_{L^{1}\left((0, T) \times F_{2}\right)}\left|h_{2, i, j}(r, y)\right| \\
& \leq \frac{27}{8}\left[c_{1}(\psi)\right]^{-3} \sigma(\partial \Omega) \sum_{i=1}^{+\infty} \kappa_{i, j}\left\|h_{1, i, j}\right\|_{L^{\infty}((0, T) \times \partial \Omega)}=: K_{3},
\end{aligned}
$$

since $s_{0} \geq 1$. We stress that, according to assumption (4.7) and definition (2.4), functions $h_{2, i, j}$ must vanish exponentially as $t \rightarrow 0+$ and $t \rightarrow T-$.

We can now state the main result of this section.

Theorem 1. Let $f \in L^{\infty}\left(Q_{T}\right), h \in L^{2}\left(Q_{T}\right), g \in H^{1}\left((0, T) ; L^{2}(\Omega)\right) \cap L^{2}((0, T)$; $\left.H^{2}(\Omega)\right), \beta \in H^{1}(0, T)$. Moreover, let $f$ and the kernels $k_{j}: E_{j} \rightarrow \mathbb{R}, j=1,2$, satisfy, respectively, conditions (4.4) and (4.5). Then the solution $u$ to problem (IP4) satisfies the weak Carleman estimate

$$
\begin{aligned}
& p\left(s_{0}, f\right) \int_{Q_{T}} l(t)^{-3} \exp \left[-2 s_{0} c_{1}(\psi) l(t)^{-1}\right]|u(t, x)|^{2} d t d x \\
& \quad+s_{0} \int_{Q_{T}} l(t)^{-1}|\nabla u(t, x)|^{2} \exp \left[2 s_{0} \alpha_{\lambda}(t, x)\right] d t d x+s_{0}^{-1} e^{-\lambda\|\psi\|_{\infty}}
\end{aligned}
$$




$$
\begin{aligned}
& \times \int_{Q_{T}} l(t)\left[\left|D_{t} u(t, x)\right|^{2}+\sum_{i, j=1}^{n}\left|D_{x_{i}} D_{x_{j}} u(t, x)\right|^{2}\right] \exp \left[2 s_{0} \alpha_{\lambda}(t, x)\right] d t d x \\
\leq & 2 C_{1} \int_{Q_{T}}|\widetilde{h}(t, x)|^{2} d t d x+2 s_{0} C_{1} \int_{\Sigma_{T}}|g(t, x)|^{2} d t d \sigma(x),
\end{aligned}
$$

where $c_{1}(\psi)=e^{2 \lambda\|\psi\|_{\infty}}$. Moreover, the solution $(u, \alpha)$ to problem (IP3), (3.2) satisfies estimate (4.9) and the following

$$
\begin{aligned}
& \int_{0}^{T}|\alpha(t)|^{2} \exp \left[-2 s_{0} c_{1}(\psi) l(t)^{-1}\right] d t \leq 2 C_{1} m^{-2} p\left(s_{0}, f\right)^{-1}\|f\|_{L^{\infty}\left(Q_{T}\right)}^{2} m(\Omega) \\
& \quad \times\left[K_{0}+\mu_{1} K_{2}\right]\left[K_{1}+\mu_{1} K_{3}\right]\left[\int_{Q_{T}}|\widetilde{h}(t, x)|^{2} d t d x\right. \\
& \left.\quad+s_{0} \int_{\Sigma_{T}}|g(t, x)|^{2} d t d \sigma(x)\right]+2 \int_{0}^{T}|I(\beta, f, g, h)(t)|^{2} d t
\end{aligned}
$$

In particular, if $(g, h, \beta)=(0,0,0)$, the linear identification problem (IP3), (3.2) admits the null solution, only.

Remark 2. As a by-product, we have proved the uniqueness of the solution to the direct ill-posed problem (IP3) with $\alpha=0$, i.e. to problem (IP4) with $\Lambda=O$ and $I=0$.

To the proof of Theorem 1 we premise two lemmata.

Lemma 1. Let $\omega=\left\{(t, r) \in(0, T)^{2}: r<t\right\}$ and $\widetilde{k}: \omega \times \Omega \rightarrow \mathbb{C}$ be a measurable function satisfying the following inequalities for some positive constants $K_{0}(\widetilde{k})$, $K_{1}(\widetilde{k})$ and $s_{0} \geq \widehat{s}_{0}$ :

$$
\begin{aligned}
& K_{0}(\widetilde{k})=\underset{t \in(0, T)}{\operatorname{ess} \sup _{Q_{t}}} \int_{Q_{t}}|\widetilde{k}(t, r, y)| d r d y<\infty, \\
& \int_{r}^{T}|\widetilde{k}(t, r, y)| d t \leq K_{1}(\widetilde{k}) l(r)^{-3} \exp \left[-2 s_{0} c_{1}(\psi) l(r)^{-1}\right], \quad(r, y) \in Q_{T},
\end{aligned}
$$

where

$$
c_{1}(\psi)=e^{2 \lambda\|\psi\|_{\infty}}-e^{\lambda \psi_{m}}, \quad \psi_{m}=\min _{x \in \bar{\Omega}} \psi(x) .
$$

Then the linear operator

$$
\widetilde{B} u(t)=\int_{Q_{t}} \widetilde{k}(t, r, y) u(r, y) d r d y, \quad t \in(0, T)
$$

satisfies the weighted inequality

$$
\begin{aligned}
& m(\Omega) \int_{0}^{T}|\widetilde{B} u(t)|^{2} \exp \left[2 s_{0} c_{1}(\psi) l(t)^{-1}\right] d t \\
& \quad \leq \int_{Q_{T}}|\widetilde{B} u(t)|^{2} \exp \left[2 s_{0} \alpha_{\lambda}(t, x)\right] d t d x \\
& \quad \leq m(\Omega) K_{0}(\widetilde{k}) K_{1}(\widetilde{k}) \int_{Q_{T}} l(t)^{-3}|v(t, x)|^{2} \exp \left[2 s_{0} \alpha_{\lambda}(t, x)\right] d t d x .
\end{aligned}
$$


Proof. Consider first the inequalities

$$
\begin{aligned}
|\widetilde{B} u(t)|^{2} & \leq \int_{Q_{t}} \widetilde{k}(t, r, y) d r d y \int_{Q_{t}}|\widetilde{k}(t, r, y)||u(r, y)|^{2} d r d y \\
& \leq K_{0}(\widetilde{k}) \int_{Q_{t}}|\widetilde{k}(t, r, y)||u(r, y)|^{2} d r d y, \quad(t, x) \in Q_{T} .
\end{aligned}
$$

Then we have the following chain of inequalities

$$
\begin{aligned}
& \int_{Q_{T}}|\widetilde{B} u(t)|^{2} \exp \left[2 s_{0} \alpha_{\lambda}(t, x)\right] d t d x \\
& \quad \leq K_{0}(\widetilde{k}) \int_{Q_{T}} \exp \left[2 s_{0} \alpha_{\lambda}(t, x)\right] d t d x \int_{Q_{t}}\left|\widetilde{k}_{\lambda}(t, r, y)\right||u(r, y)|^{2} d r d y \\
& \quad \leq K_{0}(\widetilde{k}) \int_{Q_{T}} \bar{k}_{\lambda}(r, y) l(r)^{-3} \exp \left[2 s_{0} \alpha_{\lambda}(r, y)\right]|u(r, y)|^{2} d r d y,
\end{aligned}
$$

where, for all $(r, y) \in Q_{T}$, we have set

$$
\bar{k}_{\lambda}(r, y)=\int_{(r, T) \times \Omega} l(r)^{3} \exp \left[2 s_{0}\left(\alpha_{\lambda}(t, x)-\alpha_{\lambda}(r, y)\right)\right]|\widetilde{k}(t, r, y)| d t d x .
$$

Observe now the identities

$$
\begin{aligned}
\alpha_{\lambda}(t, x)-\alpha_{\lambda}(r, y) & =\left[\alpha_{\lambda}(t, x)-\alpha_{\lambda}(r, x)\right]+\left[\alpha_{\lambda}(r, x)-\alpha_{\lambda}(r, y)\right] \\
& =\frac{l(t)-l(r)}{l(t) l(r)}\left[e^{2 \lambda\|\psi\|_{\infty}}-e^{\lambda \psi(x)}\right]+\frac{1}{l(r)}\left[e^{\lambda \psi(x)}-e^{\lambda \psi(y)}\right] .
\end{aligned}
$$

Note that, when $t>r$,

$$
l(t)-l(r)=(t-r)(T-t-r)>0
$$

if and only if $r \in(0, T / 2)$ and $t \in(r, T-r)$, so that, in this case, we have $[l(t)-l(r)][l(t) l(r)]^{-1} \leq l(r)^{-1}$. Since

$$
\begin{aligned}
e^{2 \lambda\|\psi\|_{\infty}}-e^{\lambda \psi(x)} & \leq e^{2 \lambda\|\psi\|_{\infty}}-e^{\lambda \psi_{m}}=c_{1}(\psi), \\
e^{\lambda \psi(x)}-e^{\lambda \psi(y)} & \leq e^{2 \lambda\|\psi\|_{\infty}}-e^{\lambda \psi_{m}} \leq c_{1}(\psi),
\end{aligned}
$$

we easily deduce the inequalities

$$
\begin{aligned}
\alpha_{\lambda}(t, x)-\alpha_{\lambda}(r, y) & \leq l(r)^{-1} \begin{cases}{\left[e^{2 \lambda\|\psi\|_{\infty}}-e^{\lambda \psi(y)}\right],} & \text { if } t \in(\max \{r, T-r\}, T) \\
{\left[e^{2 \lambda\|\psi\|_{\infty}}-e^{\lambda \psi(y)}\right],} & \text { otherwise }\end{cases} \\
& \leq c_{1}(\psi) l(r)^{-1}
\end{aligned}
$$

Then, according to assumption (4.11) and (4.12), for all $(r, y) \in Q_{T}$ we conclude that

$$
\begin{aligned}
\bar{k}_{\lambda}(r, y) \leq l(r)^{3} \exp \left[2 s_{0} c_{1}(\psi) l(r)^{-1}\right] \int_{(r, T) \times \Omega}|\widetilde{k}(t, r, y)| d t d x \\
\leq m(\Omega) l(r)^{3} \exp \left[2 s_{0} c_{1}(\psi) l(r)^{-1}\right] \int_{r}^{T}|\widetilde{k}(t, r, y)| d t \\
\leq m(\Omega) K_{1}(\widetilde{k}) .
\end{aligned}
$$


Finally, the latter inequality in (4.13) easily follows from (4.14), (4.19), while the first is an easy consequence of (4.16).

We prove now the following Lemma 2 concerning the kernels $k_{j}, j=1,2$.

Lemma 2. Let $E_{1, t_{1}, t_{2}}=\left(t_{1}, t_{2}\right) \times \Omega$ and $E_{2, t_{1}, t_{2}}=\left(t_{1}, t_{2}\right) \times \partial \Omega, 0 \leq t_{1}<t_{2} \leq$ $T$. Let $k_{j}: E_{j, 0, T} \times Q_{T} \rightarrow \mathbb{C}, j=1,2$, be two measurable functions satisfying inequalities (4.4) and (4.5). Then the linear operator

$$
B_{j} u(t, x)=\int_{Q_{t}} k_{1}(t, x, r, y) u(r, y) d r d y, \quad(t, x) \in E_{j, 0, T},
$$

satisfies the weighted inequality

$$
\begin{aligned}
& \int_{E_{j, 0, T}}\left|B_{j} u(t, x)\right|^{2} \exp \left[2 s_{0} \alpha_{\lambda}(t, x)\right] d t d \rho_{j}(x) \\
& \quad \leq K_{2(j-1)} K_{2 j-1} \int_{Q_{T}} l(t)^{-3}|u(t, x)|^{2} \exp \left[2 s_{0} \alpha_{\lambda}(t, x)\right] d t d \rho_{j}(x), \quad j=1,2,
\end{aligned}
$$

where $\rho_{1}=m$ and $\rho_{2}=\sigma$.

Proof. Consider first the inequalities

$$
\begin{aligned}
& \left|B_{j} u(t, x)\right|^{2} \leq \int_{Q_{t}} k_{j}(t, x, r, y) d r d y \int_{Q_{t}}\left|k_{j}(t, x, r, y)\right||u(r, y)|^{2} d r d y \\
& \quad \leq K_{2(j-1)} \int_{Q_{t}}\left|k_{j}(t, x, r, y)\right||u(r, y)|^{2} d r d y, \quad(t, x) \in E_{j, 0, T}, j=1,2 .
\end{aligned}
$$

Then we have the following chain of inequalities

$$
\begin{aligned}
& \int_{E_{j, 0, T}}\left|B_{j} u(t, x)\right|^{2} \exp \left[2 s_{0} \alpha_{\lambda}(t, x)\right] d t d \rho_{j}(x) \\
& \quad \leq K_{2(j-1)} \int_{E_{j, 0, T}} \exp \left[2 s_{0} \alpha_{\lambda}(t, x)\right] d t d \rho_{j}(x) \int_{Q_{t}}\left|k_{j, \lambda}(t, r, x, y)\right||u(r, y)|^{2} d r d y \\
& \quad \leq K_{2(j-1)} \int_{Q_{T}} \bar{k}_{j}(r, y) l(r)^{-3} \exp \left[2 s_{0} \alpha_{\lambda}(r, y)\right]|u(r, y)|^{2} d r d y
\end{aligned}
$$

where, for all $(t, x) \in E_{j, 0, T}$, we have set

$$
\bar{k}_{j, \lambda}(r, y)=\int_{E_{j, r, T}} l(r)^{3} \exp \left[2 s_{0}\left(\alpha_{\lambda}(t, x)-\alpha_{\lambda}(r, y)\right)\right]\left|k_{j}(t, x, r, y)\right| d t d \rho_{j}(x) .
$$

Now from (4.15)-(4.18) and assumptions (4.4) and (4.5), we conclude that

$$
\begin{aligned}
\bar{k}_{j}(r, y) & \leq l(r)^{3} \exp \left[2 c_{1}(\psi) l(r)^{-1}\right] \int_{E_{j, 0, T}}\left|k_{1}(t, x, r, y)\right| d t d \rho_{j}(x) \\
& \leq K_{2 j-1}, \quad(r, y) \in Q_{T} .
\end{aligned}
$$


Finally, the latter inequality in (4.13) easily follows from (4.20), (4.21), while the first easily follows from the inequality

$$
e^{\lambda\|\psi\|_{\infty}}-e^{\lambda \psi(x)} \leq e^{2 \lambda\|\psi\|_{\infty}}-e^{\lambda \psi_{m}}=c_{1}(\psi) .
$$

This concludes the proof of the lemma.

Proof of Theorem 1. From definition (3.5) and Lemmata 1 and 2 we easily deduce that $K_{0}(\widetilde{k})=m(\Omega)\left[K_{0}+\mu_{1} K_{2}\right]$ and $K_{1}(\widetilde{k})=m(\Omega)\left[K_{1}+\mu_{1} K_{3}\right]$.

Then we estimate $\Lambda(u) f$ :

$$
\begin{aligned}
& \int_{Q_{T}} \exp \left[2 s_{0} \alpha_{\lambda}(t, x)\right]|\Lambda(u)(t) f(t, x)|^{2} d t d x \\
& \leq m^{-2}\|f\|_{L^{\infty}\left(Q_{T}\right)}^{2} \int_{Q_{T}} \exp \left[2 s_{0} \alpha_{\lambda}(t, x)\right]|\widetilde{B} v(t)|^{2} d t \\
& \leq m^{-2}\|f\|_{L^{\infty}\left(Q_{T}\right)}^{2} m(\Omega)\left[K_{0}+\mu_{1} K_{2}\right]\left[K_{1}+\mu_{1} K_{3}\right] \\
& \quad \times \int_{Q_{T}} l(r)^{-3} \exp \left[2 s_{0} \alpha_{\lambda}(r, y)\right]|u(r, y)|^{2} d r d y .
\end{aligned}
$$

From Lemmata 1 and 2 and (4.23) we deduce the estimate

$$
\begin{aligned}
& \int_{Q_{T}}|\mathcal{B} v(t, x)|^{2} \exp \left[2 s_{0} \alpha_{\lambda}(t, x)\right] d t d x \\
& \quad \leq K_{4}(f) \int_{Q_{T}} l(t)^{-3}|v(t, x)|^{2} \exp \left[2 s_{0} \alpha_{\lambda}(t, x)\right] d t d x
\end{aligned}
$$

where we have set

$$
K_{4}(f)=K_{0} K_{1}+m^{-2}\|f\|_{L^{\infty}\left(Q_{T}\right)}^{2} m(\Omega)\left[K_{0}+\mu_{1} K_{2}\right]\left[K_{1}+\mu_{1} K_{3}\right] .
$$

Consequently, according to assumptions (4.2) and estimates (4.13), estimate (4.1) simplifies to the following

$$
\begin{aligned}
p\left(s_{0}, f\right) & \int_{Q_{T}} l(t)^{-3}|u(t, x)|^{2} \exp \left[2 s_{0} \alpha_{\lambda}(t, x)\right] d t d x \\
+ & s_{0} \int_{Q_{T}} l(t)^{-1}|\nabla u(t, x)|^{2} \exp \left[2 s_{0} \alpha_{\lambda}(t, x)\right] d t d x+s_{0}^{-1} e^{-\lambda\|\psi\|_{\infty}} \\
& \times \int_{Q_{T}} l(t)\left[\left|D_{t} u(t, x)\right|^{2}+\sum_{i, j=1}^{n}\left|D_{x_{i}} D_{x_{j}} u(t, x)\right|^{2}\right] \\
& \times \exp \left[2 s_{0} \alpha_{\lambda}(t, x)\right] d t d x \\
\leq & 2 C_{1} \int_{Q_{T}}|\widetilde{h}(t, x)|^{2} d t d x+2 s_{0} C_{1} \int_{\Sigma_{T}}|g(t, x)|^{2} d t d \sigma(x) .
\end{aligned}
$$

Taking advantage of the inequality

$$
\begin{aligned}
& \int_{Q_{T}} l(t)^{-3}|u(t, x)|^{2} \exp \left[2 s_{0} \alpha_{\lambda}(t, x)\right] d t d x \\
& \quad \geq \int_{Q_{T}} l(t)^{-3} \exp \left[-2 s_{0} c_{1}(\psi) l(t)^{-1}\right]|u(t, x)|^{2} d t d y
\end{aligned}
$$


from (4.24) we deduce the weak Carleman estimate (4.9).

To find out the corresponding estimate for the unknown function $\alpha$, from the representation (3.7), i.e. $\alpha(t)=-\Lambda(u)(t)-I(\beta, f, g, h)(t)$, and from Lemma 1 , with $\widetilde{B}=\Lambda(u)$, and the Carleman estimate (4.9) we easily deduce the estimates

$$
\begin{aligned}
\int_{0}^{T} & |\alpha(t)|^{2} \exp \left[-2 s_{0} c_{1}(\psi) l(t)^{-1}\right] d t \\
\leq & 2 \int_{Q_{T}}|\Lambda u(t)|^{2} \exp \left[2 s_{0} \alpha_{\lambda}(t, x)\right] d t d x \\
& +2 \int_{0}^{T}|I(\beta, f, g, h)(t)|^{2} \exp \left[2 s_{0} \alpha_{\lambda}(t, x)\right] d t \\
\leq & 2 C_{1} m^{-2} p\left(s_{0}, f\right)^{-1}\|f\|_{L^{\infty}\left(Q_{T}\right)}^{2} m(\Omega)\left[K_{0}+\mu_{1} K_{2}\right]\left[K_{1}+\mu_{1} K_{3}\right] \\
& \times\left[\int_{Q_{T}}|\widetilde{h}(t, x)|^{2} d t d x+s_{0} \int_{\Sigma_{T}}|g(t, x)|^{2} d t d \sigma(x)\right] \\
& +2 \int_{0}^{T}|I(\beta, f, g, h)(t)|^{2} d t .
\end{aligned}
$$

Observe now that $g=h=0$ in $Q_{T}$ and $\beta=0$ in $(0, T)$ imply successively $\gamma(0,0,0)=0, I(0, f, 0,0)=0$ for any $f$ satisfying $(3.6)$ and $\widetilde{h}=0$ in $Q_{T}$. Consequently, from estimates (4.9) and (4.10) we deduce $u=0$ in $Q_{T}$ and $\alpha=0$ in $(0, T)$. We have thus shown that problem (IP3), (3.2) admits at most one solution.

\section{A Continuous Dependence Result for the Solution to the Identification Problem Related to (IP3)}

The aim of this section is to estimate first the solution $u$ of the ill-posed problem (IP4) in $C\left((0, T] ; L^{2}(\Omega)\right) \cap L_{l o c}^{2}\left((0, T] ; H^{1}(\Omega)\right)$ and the related function $\alpha$ in $L_{l o c}^{2}((0, T] ; \mathbb{R})$. For this task we need that the kernels $k_{1}$ and $k_{2}$ satisfy the additional properties

$$
\begin{aligned}
K_{5} & :=\int_{Q_{T} \times Q_{T}} l(r)^{3} \exp \left[-2 s_{0} \alpha_{\lambda}(r, y)\right]\left|k_{1}(t, x, r, y)\right|^{2} d t d x d r d y<\infty, \\
K_{6} & :=\int_{\Sigma_{T} \times Q_{T}} l(r)^{3} \exp \left[-2 s_{0} \alpha_{\lambda}(r, y)\right]\left|k_{2}(t, x, r, y)\right|^{2} d t d \sigma(x) d r d y<\infty .
\end{aligned}
$$

Remark 3. Since $\exp \left[-2 s_{0} \alpha_{\lambda}(r, y)\right] \leq \exp \left[2 s_{0} c_{1}(\psi) l(r)^{-1}\right]$, it suffices to replace $K_{5}$ and $K_{6}$ by

$$
\begin{aligned}
& K_{5}^{\prime}=\int_{Q_{T} \times Q_{T}} l(r)^{3} \exp \left[2 s_{0} c_{1}(\psi) l(r)^{-1}\right]\left|k_{1}(t, x, r, y)\right|^{2} d t d x d r d y<\infty \\
& K_{6}^{\prime}=\int_{\Sigma_{T} \times Q_{T}} l(r)^{3} \exp \left[2 s_{0} c_{1}(\psi) l(r)^{-1}\right]\left|k_{2}(t, x, r, y)\right|^{2} d t d \sigma(x) d r d y<\infty .
\end{aligned}
$$


If we restrict condition (4.5) to the following

$$
\begin{gathered}
\int{ }_{-}(0, T) \times F_{j}\left|k_{j}(t, x, r, y)\right|^{2} d t d x \leq K_{2 j-1} l(r)^{-3} \exp \left[-2 s_{0} c_{1}(\psi) l(r)^{-1}\right] \\
(r, y) \in Q_{T}, j=1,2, F_{1}=\Omega, F_{2}=\partial \Omega
\end{gathered}
$$

it is easy to observe that $K_{5}^{\prime}$ and $K_{6}^{\prime}$ satisfy the inequalities

$$
\begin{aligned}
& K_{5}^{\prime} \leq K_{1} \int_{Q_{T}} d r d y=K_{1} \operatorname{Tm}(\Omega)=: K_{5}^{\prime \prime}, \\
& K_{6}^{\prime} \leq K_{3} \int_{Q_{T}} d r d y=K_{3} \operatorname{Tm}(\Omega)=: K_{6}^{\prime \prime} .
\end{aligned}
$$

Therefore, under assumptions (5.3) we can replace the pair $\left(K_{5}, K_{6}\right)$ with $\left(K_{5}^{\prime \prime}, K_{6}^{\prime \prime}\right)$. In particular, when functions $k_{j}, j=1,2$ are defined by formula (4.6), then conditions (5.3) is satisfied, if conditions (4.7) are replaced with the stricter one

$$
\left|h_{2, i, j}(r, y)\right| \leq \kappa_{i, j} l(r)^{-3 / 2} \exp \left[-s_{0} c_{1}(\psi) l(r)^{-1}\right], \quad(r, y) \in Q_{T}, j=1,2
$$

and conditions (4.8) are implemented with

$$
\sum_{i=1}^{\infty} \kappa_{i, j}^{2}<+\infty, \quad \sum_{i=1}^{+\infty}\left\|h_{1, i, j}\right\|_{L^{2}\left((0, T) \times F_{j}\right)}^{2}<+\infty, \quad j=1,2 .
$$

Indeed, we get

$$
\begin{aligned}
& \int_{(0, T) \times F_{j} \times Q_{T}} l(r)^{3} \exp \left[2 s_{0} c_{1}(\psi) l(r)^{-1}\right]\left|k_{1}(t, x, r, y)\right|^{2} d t d x d r d y \\
& \leq \int_{(0, T) \times F_{j}} \sum_{i=1}^{+\infty}\left|h_{1, i, j}(t, x)\right|^{2} d t d x \\
& \quad \times \int_{Q_{T}} \sum_{i=1}^{+\infty} l(r)^{3} \exp \left[2 s_{0} c_{1}(\psi) l(r)^{-1}\right]\left|h_{2, i, j}(r, y)\right|^{2} d r d y \\
& \leq \sum_{i=1}^{+\infty}\left\|h_{1, i, j}\right\|_{L^{2}\left((0, T) \times F_{j}\right)}^{2} \sum_{i=1}^{+\infty} \kappa_{i, j}^{2}, \quad j=1,2 .
\end{aligned}
$$

Finally, we note that conditions (4.8) are implied by the stronger (5.4).

We now state our continuous dependence theorem.

Theorem 2. Let $f \in L^{\infty}\left(Q_{T}\right), h \in L^{2}\left(Q_{T}\right), g \in H^{1}\left((0, T) ; L^{2}(\Omega)\right) \cap L^{2}((0, T)$; $\left.H^{2}(\Omega)\right), \beta \in H^{1}(0, T)$. Moreover, let $f$ and the kernels $k_{1}: Q_{T} \times Q_{T} \rightarrow \mathbb{R}$ and $k_{1}: Q_{T} \times Q_{T} \rightarrow \mathbb{R}$ satisfy, respectively, conditions (3.6) and (4.4), (4.5), (5.1), (5.2). Then the solution $(u, \alpha)$ to problem (IP3), (3.2) satisfies, for all $\varepsilon \in(0,1 / 4)$ and $\tau \in(2 \varepsilon T, T)$, the continuous dependence estimates

$$
\begin{aligned}
&\|u(\tau, \cdot)\|_{L^{2}(\Omega)}^{2}+2 \mu_{0} \int_{2 \varepsilon T}^{\tau}\|\nabla u(t, \cdot)\|_{L^{2}(\Omega)}^{2} d t \\
& \leq \Phi_{1}(\varepsilon, m, f)\left\{\|g\|_{H^{1}\left((0, T) ; L^{2}(\Omega)\right)}^{2}+\|g\|_{L^{2}\left((0, T) ; H^{2}(\Omega)\right.}^{2}\right. \\
&\left.\quad+\|h\|_{L^{2}\left(Q_{T}\right)}^{2}+\|\beta\|_{H^{1}((0, T)}^{2}\right\},
\end{aligned}
$$




$$
\begin{aligned}
\|\alpha\|_{L^{2}(0, T)} \leq & \Phi_{2}(\varepsilon, m, f)\left\{\|g\|_{H^{1}\left((0, T) ; L^{2}(\Omega)\right)}+\|g\|_{L^{2}\left((0, T) ; H^{2}(\Omega)\right.}\right. \\
& \left.+\|h\|_{L^{2}\left(Q_{T}\right)}+\|\beta\|_{H^{1}((0, T)}\right\},
\end{aligned}
$$

where $\Phi_{j}, j=1,2$, are two positive functionals depending continuously on the triplet $(\varepsilon, m, f) \in(0,1 / 2) \times \mathbb{R}_{+} \times L^{\infty}\left(Q_{T}\right)$.

Proof. First we introduce the family of functions $\sigma_{\varepsilon} \in W^{1, \infty}([0, T] ;[0,1])$, $\varepsilon \in(0,1 / 4)$, defined by

$$
\sigma_{\varepsilon}(t)= \begin{cases}0, & t \in[0, \varepsilon T], \\ (\varepsilon T)^{-1}(t-\varepsilon T), & t \in(\varepsilon T, 2 \varepsilon T), \\ 1, & t \in[2 \varepsilon T, T] .\end{cases}
$$

Introduce also the function $u_{\varepsilon}=\sigma_{\varepsilon} u$. It is a simply task to show that $u_{\varepsilon}$ solves the following initial and boundary-value problem:

$$
(\mathrm{DP} 1) \begin{cases}u_{\varepsilon} \in H^{1}\left((0, T) ; L^{2}(\Omega)\right) \cap L^{2}\left((0, T) ; H^{2}(\Omega)\right), & \\ D_{t} u_{\varepsilon}(t, x)-A(x, D) u_{\varepsilon}(t, x) & (t, x) \in Q_{T}, \\ \quad=\sigma_{\varepsilon}(t) \mathcal{B} u(t, x)+\sigma_{\varepsilon}^{\prime}(t) u(t, x)+\widetilde{h}_{\varepsilon}(t, x), & x \in \Omega, \\ u_{\varepsilon}(0, x)=0, & (t, x) \in \Sigma_{T} . \\ u_{\varepsilon}(t, x)=0, & \end{cases}
$$

Recall now that $-A(\cdot, D)$ satisfies the following estimate for all $v \in H^{2}(\Omega) \cap$ $H_{0}^{1}(\Omega)$ :

$$
\begin{aligned}
-\langle A(\cdot, D) v, v\rangle & =\int_{\Omega} \sum_{i, j=1}^{n} a_{i, j}(x) D_{x_{i}} v D_{x_{j}} v d x-\int_{\Omega} \sum_{j=1}^{n} a_{j} v D_{x_{j}} v d x \\
& =\int_{\Omega} \sum_{i, j=1}^{n} a_{i, j}(x) D_{x_{i}} v D_{x_{j}} v d x+\frac{1}{2} \int_{\Omega} v^{2} \sum_{j=1}^{n} D_{x_{j}} a_{j} d x \\
& \geq \mu_{0}\|\nabla v\|_{L^{2}(\Omega)}^{2}-\mu_{3}\|v\|_{L^{2}(\Omega)}^{2},
\end{aligned}
$$

where $\mu_{3}=\left\|\left(\sum_{j=1}^{n} D_{x_{j}} a_{j}\right)^{-}\right\|_{L^{\infty}(\Omega)} / 2, h^{-}$denoting the negative part of function $h$.

It is well-known that the solution $u_{\varepsilon}$ to (DP1) satisfies the following estimate - obtained by multiplying scalarly by $u_{\varepsilon}$ both sides in the differential equation and then integrating by parts:

$$
\begin{aligned}
& \frac{1}{2} D_{t}\left\|u_{\varepsilon}(t, \cdot)\right\|_{L^{2}(\Omega)}^{2}+\mu_{0}\left\|\nabla u_{\varepsilon}(t, \cdot)\right\|_{L^{2}(\Omega)}^{2}-\mu_{3}\left\|u_{\varepsilon}(t, \cdot)\right\|_{L^{2}(\Omega)}^{2} \\
& \quad \leq\left\|u_{\varepsilon}(t, \cdot)\right\|_{L^{2}(\Omega)}\left\{\|\mathcal{B} u(t, \cdot)\|_{L^{2}(\Omega)}+\left\|\sigma_{\varepsilon}^{\prime}(t) u(t, \cdot)\right\|_{L^{2}(\Omega)}+\left\|\widetilde{h}_{\varepsilon}(t, \cdot)\right\|_{L^{2}(\Omega)}\right\} .
\end{aligned}
$$

Integrating over $(0, \tau), \tau \in(0, T]$, we get

$$
\begin{aligned}
& \frac{1}{2}\left\|u_{\varepsilon}(\tau, \cdot)\right\|_{L^{2}(\Omega)}^{2}+\mu_{0} \int_{0}^{\tau}\left\|\nabla u_{\varepsilon}(t, \cdot)\right\|_{L^{2}(\Omega)}^{2} d t-\mu_{3} \int_{0}^{\tau}\left\|u_{\varepsilon}(t, \cdot)\right\|_{L^{2}(\Omega)}^{2} d t \\
& \leq \int_{0}^{\tau}\left\|u_{\varepsilon}(t, \cdot)\right\|_{L^{2}(\Omega)}\|\mathcal{B} u(t, \cdot)\|_{L^{2}(\Omega)} d t+\int_{0}^{\tau}\left\|u_{\varepsilon}(t, \cdot)\right\|_{L^{2}(\Omega)}\left\|\sigma_{\varepsilon}^{\prime}(t) u(t, \cdot)\right\|_{L^{2}(\Omega)} d t \\
& \quad+\int_{0}^{\tau}\left\|u_{\varepsilon}(t, \cdot)\right\|_{L^{2}(\Omega)}\left\|\widetilde{h}_{\varepsilon}(t, \cdot)\right\|_{L^{2}(\Omega)} d t .
\end{aligned}
$$


Observe then that operator $\mathcal{B}$ admits the representation

$$
\mathcal{B} u(t, x)=\int_{Q_{t}} k_{3}(t, x, r, y) u(r, y) d r d y, \quad(t, x) \in Q_{T},
$$

where the kernel $k_{3}: Q_{T} \times Q_{T} \rightarrow \mathbb{C}$ is defined, for all $(t, x, r, y) \in Q_{T} \times Q_{T}$, by

$$
k_{3}(t, x, r, y)=k_{1}(t, x, r, y)-\chi(f)(t) f(t, x) \tilde{k}(t, r, y) .
$$

Note now that from definition (3.3) and inequalities (3.6), (5.1) we deduce

$$
\begin{aligned}
\int_{Q_{T} \times Q_{T}} l(r)^{3} \exp \left[-2 s_{0} \alpha_{\lambda}(r, y)\right]|\chi(f)(t)|^{2}|f(t, x)|^{2}\left|\widetilde{k}_{1}(t, r, y)\right|^{2} d t d x d r d y \\
\leq m^{-2}\|f\|_{L^{\infty}\left(Q_{T}\right)}^{2} \int_{Q_{T} \times Q_{T}} l(r)^{3} \exp \left[-2 s_{0} \alpha_{\lambda}(r, y)\right] d t d x d r d y \\
\quad \times \int_{\Omega}\left|k_{1}(t, \xi, r, y)\right|^{2} d \xi \leq m^{-2} m(\Omega)\|f\|_{L^{\infty}\left(Q_{T}\right)}^{2} \\
\quad \times \int_{Q_{T} \times Q_{T}} l(r)^{3} \exp \left[-2 s_{0} \alpha_{\lambda}(r, y)\right]\left|k_{1}(t, \xi, r, y)\right|^{2} d t d \xi d r d y \\
\leq m^{-2} m(\Omega)\|f\|_{L^{\infty}\left(Q_{T}\right)}^{2} K_{5}
\end{aligned}
$$

Similarly, from (3.4) and (5.2) we get

$$
\begin{aligned}
& \int_{\Sigma_{T} \times Q_{T}} l(r)^{3} \exp \left[-2 s_{0} \alpha_{\lambda}(r, y)\right]|\chi(f)(t)|^{2}|f(t, x)|^{2}\left|\tilde{k}_{2}(t, r, y)\right|^{2} d t d \sigma(x) d r d y \\
& \leq m^{-2}\|f\|_{L^{\infty}\left(Q_{T}\right)}^{2} \int_{\Sigma_{T} \times Q_{T}} l(r)^{3} \exp \left[-2 s_{0} \alpha_{\lambda}(r, y)\right] d t d \sigma(x) d r d y \\
& \quad \times \int_{\partial \Omega} \bar{\nu}_{A}(\xi)\left|k_{2}(t, \xi, r, y)\right|^{2} d \xi \leq m^{-2} \mu_{1} \sigma(\partial \Omega)\|f\|_{L^{\infty}\left(Q_{T}\right)}^{2} \\
& \quad \times \int_{\Sigma_{T} \times Q_{T}} l(r)^{3} \exp \left[-2 s_{0} \alpha_{\lambda}(r, y)\right]\left|k_{2}(t, \xi, r, y)\right|^{2} d t d \sigma(\xi) d r d y \\
& \leq m^{-2} \mu_{1} \sigma(\partial \Omega)\|f\|_{L^{\infty}\left(Q_{T}\right)}^{2} K_{6} .
\end{aligned}
$$

Hence, from definitions (5.10), (3.3) and (3.4) we deduce the estimate

$$
\begin{aligned}
\int_{Q_{T} \times Q_{T}} l(r)^{3} \exp \left[-2 s_{0} \alpha_{\lambda}(r, y)\right]\left|k_{3}(t, x, r, y)\right|^{2} d t d x d r d y \\
\leq 3 \int_{Q_{T} \times Q_{T}} l(r)^{3} \exp \left[-2 s_{0} \alpha_{\lambda}(r, y)\right] \\
\quad \times\left[\left|k_{1}(t, x, r, y)\right|^{2}+m^{-2}|f(t, x)|^{2} \sum_{j=1}^{2}\left|\widetilde{k}_{2}(t, r, y)\right|^{2}\right] d t d x d r d y \\
\leq\left\{3 K_{5}+3 m^{-2}\left[m(\Omega) K_{5}+\mu_{1} \sigma(\partial \Omega) K_{6}\right]\|f\|_{L^{\infty}\left(Q_{T}\right)}^{2}\right\}=: K_{7}(m, f) .
\end{aligned}
$$

Consequently, using the Carleman estimate (4.9), we deduce the following weighted estimate for $\mathcal{B} u$, holding for all $\tau \in(0, T)$ :

$$
\int_{Q_{\tau}}|\mathcal{B} u(t, x)|^{2} d t d x \leq \int_{Q_{\tau}}\left[\int_{Q_{t}} l(r)^{3 / 2} \exp \left[-s_{0} \alpha_{\lambda}(r, y)\right]\right.
$$




$$
\begin{aligned}
\times & \left.\left|k_{3}(t, x, r, y)\right| l(r)^{-3 / 2} \exp \left[s_{0} \alpha_{\lambda}(r, y)\right]|u(r, y)| d r d y\right]^{2} d t d x \\
\leq & \int_{Q_{\tau}}\left[\int_{Q_{t}} l(r)^{3} \exp \left[-2 s_{0} \alpha_{\lambda}(r, y)\right]\left|k_{3}(t, x, r, y)\right|^{2} d r d y\right. \\
& \left.\times \int_{Q_{t}} l(r)^{-3} \exp \left[2 s_{0} \alpha_{\lambda}(r, y)\right]|u(r, y)|^{2} d r d y\right] d t d x \\
\leq & \int_{Q_{\tau} \times Q_{t}} l(r)^{3} \exp \left[-2 s_{0} \alpha_{\lambda}(r, y)\right]\left|k_{3}(t, x, r, y)\right|^{2} d t d x d r d y \\
& \times \int_{Q_{T}} l(r)^{-3} \exp \left[2 s_{0} \alpha_{\lambda}(r, y)\right]|u(r, y)|^{2} d r d y \\
\leq & 2 C_{1} K_{7}(m, f) p\left(s_{0}, f\right)^{-1}\left[\|\widetilde{h}\|_{L^{2}\left(Q_{T}\right)}^{2}+\|g\|_{L^{2}\left(\Sigma_{T}\right)}^{2}\right] \\
= & : J_{1}(m, f, g, \widetilde{h}) .
\end{aligned}
$$

Finally, we have

$$
\begin{aligned}
& \int_{0}^{\tau}\left\|u_{\varepsilon}(t, \cdot)\right\|_{L^{2}(\Omega)}\|\mathcal{B} u(t, \cdot)\|_{L^{2}(\Omega)} d t \\
& \quad \leq \frac{1}{2} \int_{0}^{\tau}\left\|u_{\varepsilon}(t, \cdot)\right\|_{L^{2}(\Omega)}^{2} d t+\frac{1}{2} \int_{Q_{\tau}}|\mathcal{B} u(t, x)|^{2} d t d x \\
& \quad \leq \frac{1}{2} \int_{0}^{\tau}\left\|u_{\varepsilon}(t, \cdot)\right\|_{L^{2}(\Omega)}^{2} d t+\frac{1}{2} J_{1}(m, f, g, \widetilde{h}) .
\end{aligned}
$$

Using now the inclusion $\operatorname{supp} \sigma_{\varepsilon}^{\prime} \subset[\varepsilon T, 2 \varepsilon T]$, we get the inequalities

$$
\begin{aligned}
& \int_{0}^{\tau}\left|\sigma_{\varepsilon}^{\prime}(t)\right|\left\|u_{\varepsilon}(t, \cdot)\right\|_{L^{2}(\Omega)}\|u(t, \cdot)\|_{L^{2}(\Omega)} d t \\
& \quad \leq \frac{1}{2} \int_{0}^{\tau}\left\|u_{\varepsilon}(t, \cdot)\right\|_{L^{2}(\Omega)}^{2} d t+\frac{1}{2} \int_{0}^{\tau}\left|\sigma_{\varepsilon}^{\prime}(t)\right|^{2}\|u(t, \cdot)\|_{L^{2}(\Omega)}^{2} d t \\
& \quad \leq \frac{1}{2} \int_{0}^{\tau}\left\|u_{\varepsilon}(t, \cdot)\right\|_{L^{2}(\Omega)}^{2} d t+\frac{1}{2} \int_{0}^{T}\left|\sigma_{\varepsilon}^{\prime}(t)\right|^{2}\|u(t, \cdot)\|_{L^{2}(\Omega)}^{2} d t \\
& \quad \leq \frac{1}{2} \int_{0}^{\tau}\left\|u_{\varepsilon}(t, \cdot)\right\|_{L^{2}(\Omega)}^{2} d t+\frac{1}{2}\left\|\sigma_{\varepsilon}^{\prime}\right\|_{L^{\infty}(0, T)}^{2} \int_{\varepsilon T}^{2 \varepsilon T}\|u(t, \cdot)\|_{L^{2}(\Omega)}^{2} d t .
\end{aligned}
$$

Observe now that

$$
\begin{aligned}
\rho_{1, \lambda}\left(t, s_{0}\right) & :=\exp \left\{-2 s_{0}\left[e^{2 \lambda\|\psi\|_{\infty}}-e^{\lambda \psi_{m}}\right] l(t)^{-1}\right\} \leq \exp \left[2 s_{0} \alpha_{\lambda}(t, x)\right] \quad(5.16) \\
& \leq \exp \left\{-2 s_{0}\left[e^{2 \lambda\|\psi\|_{\infty}}-e^{\lambda\|\psi\|_{\infty}}\right] l(t)^{-1}\right\}=: \rho_{2, \lambda}\left(t, s_{0}\right), \quad(t, x) \in Q_{T},
\end{aligned}
$$

where $\psi_{m}=\min _{x \in \bar{\Omega}} \psi(x)$. From (4.24), (5.7) and from the inequality

$$
\begin{aligned}
1 & =l(t)^{3} \rho_{1, \lambda}\left(t, s_{0}\right)^{-1} l(t)^{-3} \rho_{1, \lambda}\left(t, s_{0}\right) \leq 2^{-6} T^{6} \rho_{1, \lambda}\left(\varepsilon T, s_{0}\right)^{-1} l(t)^{-3} \rho_{1, \lambda}\left(t, s_{0}\right) \\
& =: C_{2}\left(s_{0}, \varepsilon, T\right) l(t)^{-3} \rho_{1, \lambda}\left(t, s_{0}\right), \quad t \in[\varepsilon T, 2 \varepsilon T], \varepsilon \in(0,1 / 2)
\end{aligned}
$$

we get

$$
\int_{\varepsilon T}^{2 \varepsilon T}\|u(t, \cdot)\|_{L^{2}(\Omega)}^{2} d t \leq C_{2}\left(s_{0}, \varepsilon, T\right) \int_{\varepsilon T}^{2 \varepsilon T} l(t)^{-3} \rho_{1, \lambda}\left(t, s_{0}\right)\|u(t, \cdot)\|_{L^{2}(\Omega)}^{2} d t
$$




$$
\begin{aligned}
\leq & C_{2}\left(s_{0}, \varepsilon, T\right) \int_{0}^{T} l(t)^{-3} \rho_{1, \lambda}\left(t, s_{0}\right)\|u(t, \cdot)\|_{L^{2}(\Omega)}^{2} d t \\
\leq & 3 C_{1} C_{2}\left(s_{0}, \varepsilon, T\right) p_{0}\left(s_{0}, f\right)^{-1} \\
& \times \int_{Q_{T}}|\widetilde{h}(t, x)|^{2} \exp \left[2 s_{0} \alpha_{\lambda}(t, x)\right] d t d x \\
\leq & 3 C_{1} C_{2}\left(s_{0}, \varepsilon, T\right) p_{0}\left(s_{0}, f\right)^{-1}\left[\|\widetilde{h}\|_{L^{2}\left(Q_{T}\right)}^{2}+\|g\|_{L^{2}\left(\Sigma_{T}\right)}^{2}\right] \\
= & : J_{2}(\varepsilon, m, f, g, \widetilde{h}) .
\end{aligned}
$$

Therefore, taking (4.9) and (5.14), (5.15), (5.17) into account, from (5.8) we easily deduce, for all $\tau \in(0, T)$, the following integral inequality:

$$
\begin{aligned}
\left\|u_{\varepsilon}(\tau, \cdot)\right\|_{L^{2}(\Omega)}^{2}+2 \mu_{0} \int_{0}^{\tau}\left\|\nabla u_{\varepsilon}(t, \cdot)\right\|_{L^{2}(\Omega)}^{2} d t \\
\leq 2 \int_{0}^{\tau}\left\|u_{\varepsilon}(t, \cdot)\right\|_{L^{2}(\Omega)}^{2} d t+2 \int_{0}^{\tau}\left\|\widetilde{h}_{\varepsilon}(t, \cdot)\right\|_{L^{2}(\Omega)}\left\|u_{\varepsilon}(t, \cdot)\right\|_{L^{2}(\Omega)} d t \\
\quad+(\varepsilon T)^{-2} \int_{\varepsilon T}^{2 \varepsilon T}\|u(t, \cdot)\|_{L^{2}(\Omega)}^{2} d t+J_{1}(m, f, g, \widetilde{h}) . \\
\leq 2 \int_{0}^{\tau}\left\|u_{\varepsilon}(t, \cdot)\right\|_{L^{2}(\Omega)}^{2} d t+2 \int_{0}^{\tau}\left\|\widetilde{h}_{\varepsilon}(t, \cdot)\right\|_{L^{2}(\Omega)}\left\|u_{\varepsilon}(t, \cdot)\right\|_{L^{2}(\Omega)} d t \\
\quad+J(\varepsilon, m, f, \widetilde{h}),
\end{aligned}
$$

where we have set

$$
J(\varepsilon, m, f, \widetilde{h})=J_{1}(m, f, g, \widetilde{h})+(\varepsilon T)^{-2} J_{2}(\varepsilon, m, f, g, \widetilde{h}) .
$$

Finally, from (5.18) and (5.17) we deduce the fundamental integro-differential inequality

$$
\begin{aligned}
z_{\varepsilon}(\tau) & =:\left\|u_{\varepsilon}(\tau, \cdot)\right\|_{L^{2}(\Omega)}^{2}+2 \mu_{0} \int_{0}^{\tau}\left\|\nabla u_{\varepsilon}(t, \cdot)\right\|_{L^{2}(\Omega)}^{2} d t \\
& \leq J(\varepsilon, m, f, \tilde{h})+2 \int_{0}^{\tau} z_{\varepsilon}(t) d t+2 \int_{0}^{\tau}\left\|\widetilde{h}_{\varepsilon}(t, \cdot)\right\|_{L^{2}(\Omega)} z_{\varepsilon}(t)^{1 / 2} d t
\end{aligned}
$$

Then we need Theorem 4.9 in [2], with $p=1 / 2$, which we report here as a lemma.

Lemma 3. Let $z$ be a nonnegative $C([0, T])$-function and let $b, k$ be nonnegative $L^{1}((0, T))$-functions satisfying

$$
z(t) \leq a+\int_{0}^{t} b(s) z(s) d s+\int_{0}^{t} k(s) z(s)^{1 / 2} d s, \quad t \in[0, T],
$$

where $a \geq 0$ is a given constant. Then for all $t \in[0, T]$

$$
z(t) \leq \exp \left(\int_{0}^{t} b(s) d s\right)\left[a^{1 / 2}+\frac{1}{2} \int_{0}^{t} k(s) \exp \left(-\frac{1}{2} \int_{0}^{s} b(\sigma) d \sigma\right) d s\right]^{2} .
$$


Then from estimate (5.20), Lemma 3 and the elementary inequality $(a+$ $b)^{2} \leq 2 a^{2}+2 b^{2}$, for $\tau \in[0, T]$, we deduce the estimate

$$
\begin{aligned}
& \left\|u_{\varepsilon}(\tau, \cdot)\right\|_{L^{2}(\Omega)}^{2}+2 \mu_{0} \int_{0}^{\tau}\left\|\nabla u_{\varepsilon}(t, \cdot)\right\|_{L^{2}(\Omega)}^{2} d t \\
& =z_{\varepsilon}(\tau) \leq 2 J(\varepsilon, m, f, g, \widetilde{h}) \exp (2 \tau)+\left\{2 \int_{0}^{\tau} \exp (\tau-t)\|\widetilde{h}(t, \cdot)\|_{L^{2}(\Omega)} d t\right\}^{2} .
\end{aligned}
$$

Observe now that

$$
\begin{aligned}
\|\widetilde{h}\|_{L^{2}\left(Q_{T}\right)} \leq & C_{3}(\Omega, T, m)\left\{\|g\|_{H^{1}\left((0, T) ; L^{2}(\Omega)\right)}+\|g\|_{L^{2}\left((0, T) ; H^{2}(\Omega)\right)}\right. \\
& \left.+\|h\|_{L^{2}\left(Q_{T}\right)}+\|\beta\|_{H^{1}((0, T)}\right\} .
\end{aligned}
$$

Then from (5.21) and (5.22) we easily deduce the estimate

$$
\begin{aligned}
\left\|u_{\varepsilon}(\tau, \cdot)\right\|_{L^{2}(\Omega)}^{2}+2 \mu_{0} \int_{0}^{\tau}\left\|\nabla u_{\varepsilon}(t, \cdot)\right\|_{L^{2}(\Omega)}^{2} d t \\
\leq C_{4}(\varepsilon, m, f)\left\{\|g\|_{H^{1}\left((0, T) ; L^{2}(\Omega)\right)}^{2}+\|g\|_{L^{2}\left((0, T) ; H^{2}(\Omega)\right.}^{2}\right. \\
\left.\quad+\|h\|_{L^{2}\left(Q_{T}\right)}^{2}+\|\beta\|_{H^{1}((0, T)}^{2}\right\} .
\end{aligned}
$$

Taking now $\tau \in[2 \varepsilon T, T]$, from (5.23) we obtain the desired estimate (5.5) for $u$.

Then we observe that reasoning as for the proof of estimates (5.11) and (5.12), both with $f \equiv 1$, we get

$$
\begin{aligned}
& \int_{(0, T) \times Q_{T}} l(r)^{3} \exp \left[-2 s_{0} \alpha_{\lambda}(r, y)\right]\left|\widetilde{k}_{1}(t, r, y)\right|^{2} d t d r d y \\
& \quad \leq m(\Omega) \int_{(0, T) \times Q_{T}} l(r)^{3} \exp \left[-2 s_{0} \alpha_{\lambda}(r, y)\right] d t d r d y \int_{\Omega}\left|k_{1}(t, \xi, r, y)\right|^{2} d \xi \\
& \quad \leq m(\Omega) \int_{Q_{T} \times Q_{T}} l(r)^{3} \exp \left[-2 s_{0} \alpha_{\lambda}(r, y)\right]\left|k_{1}(t, \xi, r, y)\right|^{2} d t d \xi d r d y \\
& \quad \leq m(\Omega) K_{5} .
\end{aligned}
$$

Similarly we get

$$
\begin{aligned}
& \int_{(0, T) \times Q_{T}} l(r)^{3} \exp \left[-2 s_{0} \alpha_{\lambda}(r, y)\right]\left|\widetilde{k}_{2}(t, r, y)\right|^{2} d t d r d y \\
& \leq \sigma(\partial \Omega) \int_{\Sigma_{T} \times Q_{T}} l(r)^{3} \exp \left[-2 s_{0} \alpha_{\lambda}(r, y)\right] d t d \sigma(x) d r d y \int_{\partial \Omega} \bar{\nu}_{A}(\xi)\left|k_{2}(t, \xi, r, y)\right|^{2} d \xi \\
& \quad \leq \mu_{1} \sigma(\partial \Omega) \int_{\Sigma_{T} \times Q_{T}} l(r)^{3} \exp \left[-2 s_{0} \alpha_{\lambda}(r, y)\right]\left|k_{2}(t, \xi, r, y)\right|^{2} d t d \sigma(\xi) d r d y \\
& \leq \mu_{1} \sigma(\partial \Omega) K_{6} .
\end{aligned}
$$

Hence, from definitions (5.9), (3.3) and (3.4) we deduce the estimate

$$
\begin{aligned}
& \int_{(0, T) \times Q_{T}} l(r)^{3} \exp \left[-2 s_{0} \alpha_{\lambda}(r, y)\right]|\widetilde{k}(t, r, y)|^{2} d t d r d y \\
& \quad \leq 2 \int_{(0, T) \times Q_{T}} l(r)^{3} \exp \left[-2 s_{0} \alpha_{\lambda}(r, y)\right] \sum_{j=1}^{2}\left|\widetilde{k}_{j}(t, r, y)\right|^{2} d t d r d y \\
& \quad \leq 2\left(m(\Omega) K_{5}+\mu_{1} \sigma(\partial \Omega) K_{6}\right)=: K_{8} .
\end{aligned}
$$


Therefore we obtain the estimate

$$
\begin{aligned}
\int_{0}^{T}|\Lambda u(t)|^{2} d t \leq & m^{-2} \int_{0}^{T}\left\{\int_{Q_{t}} l(r)^{3 / 2} \exp \left[-s_{0} \alpha_{\lambda}(r, y)\right]|\widetilde{k}(t, r, y)|\right. \\
& \left.\times l(r)^{-3 / 2} \exp \left[s_{0} \alpha_{\lambda}(r, y)\right]|u(r, y)| d r d y\right\}^{2} d t \\
\leq & m^{-2} \int_{0}^{T} d t \int_{Q_{t}} l(r)^{3} \exp \left[-2 s_{0} \alpha_{\lambda}(r, y)\right]|\widetilde{k}(t, r, y)|^{2} d r d y \\
& \times \int_{Q_{t}} l(r)^{-3} \exp \left[2 s_{0} \alpha_{\lambda}(r, y)\right]|u(r, y)|^{2} d r d y \\
\leq & m^{-2} K_{8} p_{0}\left(s_{0}, f\right)^{-1}\left[\|\widetilde{h}\|_{L^{2}\left(Q_{T}\right)}^{2}+\|g\|_{L^{2}\left(\Sigma_{T}\right)}^{2}\right]
\end{aligned}
$$

Then from (3.7) we easily obtain the final estimate (5.6) for $\alpha$.

\section{References}

[1] E.A. Artyukhin and A.S. Okhapkin. Determination of the parameters in the generalized heat-conduction equation from transient experimental data. J. Eng. Phys. Thermophys., 42:693-698, 1982.

[2] D. Bainov and P. Simeonov. Integral Inequalities and Applications. Kluwer, 1992.

[3] J.R. Cannon. Determination of certain parameters in heat conduction problems. J. Math. Anal. Appl., 8:188-201, 1964. http://dx.doi.org/10.1016/0022-247X(64)90061-7.

[4] M. Choulli. Une introduction aux problèms inverses elliptiques et paraboliques, volume 65 of Math. and Appl. Springer-Verlag, Berlin Heidelberg, 2009.

[5] A.V. Fursikov and O.Yu. Imanuvilov. Controllability of Evolution Equations. Lecture Notes Series. Seoul National Univ., 1996.

[6] O.Yu. Imanuvilov and M. Yamamoto. Lipschitz stability in inverse parabolic problems by the Carleman estimate. Inverse Problems, 14:1229-1245, 1998. http://dx.doi.org/10.1088/0266-5611/14/5/009.

[7] A. Lorenzi. Recovering two constants in a parabolic linear equation. J. Phys. Conf. Ser., 73, 2007. http://dx.doi.org/10.1088/1742-6596/73/1/012014.

[8] A. Lorenzi. Recovering a constant in a strongly ill-posed parabolic problem. $J$. Abstr. Differ. Equ. Appl., 2(2):72-92, 2012.

[9] A. Lorenzi. Addendum to "Recovering a constant in a strongly ill-posed integrodifferential parabolic problem". J. Abstr. Differ. Equ. Appl., 3(2):17-24, 2012.

[10] A. Lorenzi and G. Mola. Identification of a real constant in linear evolution equations in Hilbert spaces. Inverse Probl. Imaging, 5:695-714, 2011.

[11] L. Lorenzi. An identification problem for the Ornstein-Uhlenbeck operator. $J$. Inverse Ill-Posed Probl., 19:293-326, 2011. http://dx.doi.org/10.1515/jiip.2011.032.

[12] G. Mola. Identification of the diffusion coefficient in linear evolution equations in Hilbert spaces. J. Abstr. Differ. Equ. Appl., 2(1):14-28, 2011.

[13] M. Yamamoto. Determination of constant parameters in some semilinear parabolic equations. In Ill-Posed Problems in Natural Sciences (Moscow, 1991), pp. 439-445, VSP, 1992. Utrecht. 